\title{
Determination of the muon charge sign with the dipolar spectrometers of the OPERA experiment
}

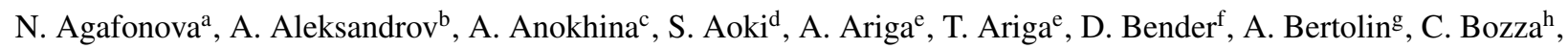
R. Brugnera ${ }^{\mathrm{i}, \mathrm{g}}$, A. Buonaura ${ }^{\mathrm{b}, \mathrm{j}}$, S. Buontempo ${ }^{\mathrm{b}}$, B. Büttner ${ }^{\mathrm{k}}$, M. Chernyavsky ${ }^{1}$, A. Chukanov ${ }^{\mathrm{m}}$, L. Consiglio ${ }^{\mathrm{b}}$, N. D' Ambrosion $^{\mathrm{n}}$, G. De Lellis ${ }^{\mathrm{b}, \mathrm{j}}$, M. De Serio ${ }^{\text {o,p }}$, P. Del Amo Sanchez ${ }^{\mathrm{q}}$, A. Di Crescenzo ${ }^{\mathrm{b}}$, D. Di Ferdinando ${ }^{\mathrm{r}}$, N. Di Marco ${ }^{\mathrm{n}}$, S. Dmitrievski ${ }^{\mathrm{m}}$,

M. Dracos ${ }^{\mathrm{s}}$, D. Duchesneau ${ }^{\mathrm{q}}$, S. Dusini ${ }^{\mathrm{g}}$, T. Dzhatdoev ${ }^{\mathrm{c}}$, J. Ebert ${ }^{\mathrm{k}}$, A. Ereditato ${ }^{\mathrm{e}}$, R. A. Fini ${ }^{\mathrm{o}}$, T. Fukudat ${ }^{\mathrm{t}}$, G. Galati ${ }^{\mathrm{o}}$,

A. Garfagnini i, ${ }^{\mathrm{i},}$, G. Giacomelli ${ }^{\mathrm{i}, \mathrm{r}, 6}$, C. Göllnitz ${ }^{\mathrm{k}}$, J. Goldberg ${ }^{\mathrm{v}}$, D. Goloubkov ${ }^{\mathrm{w}}$, Y. Gornushkin ${ }^{\mathrm{m}}$, G. Grella ${ }^{\mathrm{h}}$, M. Guler ${ }^{\mathrm{f}}$,

C. Gustavino ${ }^{\mathrm{x}}$, C. Hagner ${ }^{\mathrm{k}}$, T. Hara ${ }^{\mathrm{d}}$, A. Hollnagel ${ }^{\mathrm{k}}$, B. Hosseini ${ }^{\mathrm{b}, \mathrm{j}}$, H. Ishidat ${ }^{\mathrm{t}}$ K. Ishiguro ${ }^{\mathrm{y}}$, K. Jakovcic ${ }^{\mathrm{z}}$, C. Jollet ${ }^{\mathrm{s}}$,

C. Kamiscioglu f,aa , M. Kamiscioglu ${ }^{\mathrm{f}}$, J. Kawada ${ }^{\mathrm{e}}$, J. H. Kim ${ }^{\mathrm{ab}}$, S. H. Kim ${ }^{\mathrm{ab}, 2}$, N. Kitagawa ${ }^{\mathrm{y}}$, B. Klicek ${ }^{\mathrm{z}}$, K. Kodama ${ }^{\text {ac }}$,

M. Komatsu ${ }^{\mathrm{y}}$, U. Kose ${ }^{\mathrm{g}, 1}$, I. Kreslo ${ }^{\mathrm{e}}$, A. Lauria ${ }^{\mathrm{b}, \mathrm{j}}$, J. Lenkeit ${ }^{\mathrm{k}}$, A. Ljubicic ${ }^{\mathrm{z}}$, A. Longhin ${ }^{\mathrm{ad}}$, P. Loverre ${ }^{\mathrm{ae}, \mathrm{x}}$, A. Malgin ${ }^{\mathrm{a}}$,

M. Malenica ${ }^{\mathrm{z}}$, G. Mandrioli ${ }^{\mathrm{r}}$, T. Matsuo ${ }^{\mathrm{t}}$, V. Matveev ${ }^{\mathrm{a}}$, N. Mauri ${ }^{\mathrm{u}, \mathrm{r}}$, E. Medinaceli ${ }^{\mathrm{i}, \mathrm{g}}$, A. Meregaglia ${ }^{\mathrm{s}}$, M. Meyer ${ }^{\mathrm{k}, 1, *}, \mathrm{~S}_{\text {. Mikado }}^{\mathrm{t}}$,

P. Monacelliaf , M. C. Montesi ${ }^{\mathrm{bj}}$, K. Morishima ${ }^{\mathrm{y}}$, M. T. Muciaccia ${ }^{\mathrm{o}, \mathrm{p}}$, N. Naganawa ${ }^{\mathrm{y}}$, T. Naka ${ }^{\mathrm{y}}$, M. Nakamura ${ }^{\mathrm{y}}$, T. Nakano ${ }^{\mathrm{y}}$,

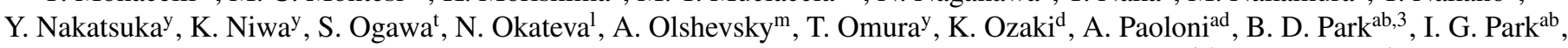
L. Pasqualini ${ }^{\mathrm{u}, \mathrm{r}}$, A. Pastore ${ }^{\mathrm{o}}$, L. Patriziir ${ }^{\mathrm{r}}$, H. Pessard ${ }^{\mathrm{q}}$, C. Pistillo ${ }^{\mathrm{e}}$, D. Podgrudkov ${ }^{\mathrm{c}}$, N. Polukhina ${ }^{1,5}$, M. Pozzato ${ }^{\mathrm{u}, 4}$, F. Pupilli $^{\mathrm{n}}$, M. Roda ${ }^{\mathrm{i}, \mathrm{g}}$, H. Rokujo ${ }^{\mathrm{y}}$, T. Roganova ${ }^{\mathrm{c}}$, G. Rosa ${ }^{\mathrm{ae}, \mathrm{x}}$, I. Rostovtseva ${ }^{\mathrm{w}}$, O. Ryazhskaya ${ }^{\mathrm{a}}$, O. Sato , $^{\mathrm{y}}$, Y. Sato ${ }^{\mathrm{ag}}$, A. Schembri ${ }^{\mathrm{n}}$,

I. Shakiryanova ${ }^{a}$, T. Shchedrina ${ }^{\mathrm{b}}$, A. Sheshukov ${ }^{\mathrm{b}}$, H. Shibuya ${ }^{\mathrm{t}}$, T. Shiraishi ${ }^{\mathrm{y}}$, G. Shoziyoev ${ }^{\mathrm{c}}$, S. Simone ${ }^{\mathrm{o}, \mathrm{p}}$, M. Sioli ${ }^{\mathrm{u}, \mathrm{r}}$,

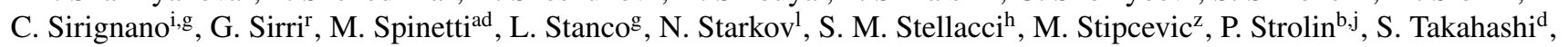
M. Tenti ${ }^{\mathrm{r}}$, F. Terranova ${ }^{\text {ad,ah }}$, V. Tioukov ${ }^{\mathrm{b}}$, S. Tufanli ${ }^{\mathrm{e}}$, P. Vilain ${ }^{\mathrm{ai}}$, M. Vladimirov ${ }^{\mathrm{l}}$, L. Votano ${ }^{\text {ad }}$, J. L. Vuilleumier ${ }^{\mathrm{e}}$, G. Wilquet ${ }^{\mathrm{ai}}$, B. Wonsak ${ }^{\mathrm{k}, *}$, C. S. Yoon ${ }^{\text {ab }}$, Y. Zaitsev ${ }^{\mathrm{w}}$, S. Zemskova ${ }^{\mathrm{m}}$, A. Zghiche ${ }^{\mathrm{q}}$

\author{
${ }^{a}$ INR Institute for Nuclear Research, Russian Academy of Sciences RUS-117312, Moscow, Russia \\ ${ }^{b}$ INFN Sezione di Napoli, I-80125 Napoli, Italy \\ ${ }^{c}$ SINP MSU-Skobeltsyn Institute of Nuclear Physics, Lomonosov Moscow State University, RUS-119992 Moscow, Russia \\ ${ }^{d}$ Kobe University, J-657-8501 Kobe, Japan \\ ${ }^{e}$ Albert Einstein Center for Fundamental Physics, Laboratory for High Energy Physics (LHEP), University of Bern, CH-3012 Bern, Switzerland \\ ${ }^{f}$ METU Middle East Technical University, TR-06531 Ankara, Turkey \\ ${ }^{g}$ INFN Sezione di Padova, I-35131 Padova, Italy \\ ${ }^{h}$ Dip. di Fisica dell'Uni. di Salerno and "Gruppo Collegato" INFN, I-84084 Fisciano (SA) Italy \\ ${ }^{i}$ Dipartimento di Fisica dell' Università di Padova, I-35131 Padova, Italy \\ ${ }^{j}$ Dipartimento di Scienze Fisiche dell'Università Federico II di Napoli, I-80125 Napoli, Italy \\ ${ }^{k}$ Hamburg University, D-22761 Hamburg, Germany \\ ${ }^{l}$ LPI-Lebedev Physical Institute of the Russian Academy of Sciences, 119991 Moscow, Russia \\ ${ }^{m}$ JINR-Joint Institute for Nuclear Research, RUS-141980 Dubna, Russia \\ ${ }^{n}$ INFN-Laboratori Nazionali del Gran Sasso, I-67010 Assergi (L'Aquila), Italy \\ ${ }^{\circ}$ INFN Sezione di Bari, I-70126 Bari, Italy \\ ${ }^{p}$ Dipartimento di Fisica dell'Università di Bari, I-70126 Bari, Italy \\ ${ }^{q}$ LAPP, Université Savoie Mont Blanc, CNRS/IN2P3, F-74941 Annecy-le-Vieux, France \\ ${ }^{r}$ INFN Sezione di Bologna, I-40127 Bologna, Italy \\ ${ }^{s} I P H C$, Université de Strasbourg, CNRS/IN2P3, F-67037 Strasbourg, France \\ ${ }^{t}$ Toho University, J-274-8510 Funabashi, Japan \\ ${ }^{u}$ Dipartimento di Fisica e Astronomia dell'Università di Bologna, I-40127 Bologna, Italy \\ ${ }^{v}$ Department of Physics, Technion, IL-32000 Haifa, Israel \\ ${ }^{w}$ ITEP-Institute for Theoretical and Experimental Physics, RUS-317259 Moscow, Russia \\ ${ }^{x}$ INFN Sezione di Roma, I-00185 Roma, Italy \\ ${ }^{y}$ Nagoya University, J-464-8602 Nagoya, Japan \\ ${ }^{z}$ IRB-Rudjer Boskovic Institute, HR-10002 Zagreb, Croatia \\ ${ }^{a a}$ Ankara University, TR-06100 Ankara, Turkey \\ ${ }^{a b}$ Gyeongsang National University, ROK-900 Gazwa-dong, Jinju 660-701, Korea \\ ${ }^{a c}$ Aichi University of Education, J-448-8542 Kariya (Aichi-Ken), Japan \\ ${ }^{a d}$ INFN-Laboratori Nazionali di Frascati dell'INFN, I-00044 Frascati (Roma), Italy \\ ${ }^{a e}$ Dipartimento di Fisica dell'Università di Roma 'La Sapienza' and INFN, I-00185 Roma, Italy
}

\footnotetext{
${ }^{*}$ Corresponding authors

Universität Hamburg, Luruper Chaussee 149, 22761 Hamburg, Germany

Email addresses: mikko.meyer@desy.de (M. Meyer), bwonsak@mail. desy.de (B. Wonsak)

${ }^{1}$ Now at CERN, Geneva, Switzerland

${ }^{2}$ Now at CUP, Institute for Basic Science, Daejeon, Korea.

${ }^{3}$ Now at Samsung Changwon Hospital, SKKU, Changwon, Korea.

${ }^{4}$ Now at Dipartimento di Fisica, Università degli Studi di Trento

${ }^{5}$ Also at National Research Nuclear University MEPhI

${ }^{6}$ deceased
} 


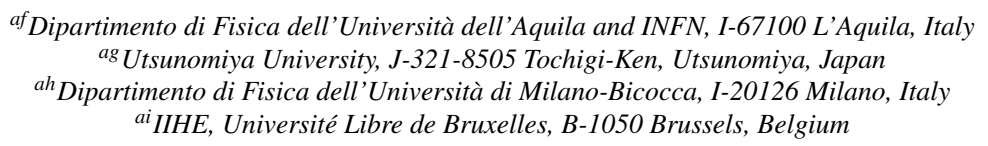

\begin{abstract}
The OPERA long-baseline neutrino-oscillation experiment has observed the direct appearance of $v_{\tau}$ in the CNGS $v_{\mu}$ beam. Two large muon magnetic spectrometers are used to identify muons produced in the $\tau$ leptonic decay and in $v_{\mu} \mathrm{CC}$ interactions by measuring their charge and momentum. Besides the kinematic analysis of the $\tau$ decays, background resulting from the decay of charmed particles produced in $v_{\mu} \mathrm{CC}$ interactions is reduced by efficiently identifying the muon track. A new method for the charge sign determination has been applied, via a weighted angular matching of the straight track-segments reconstructed in the different parts of the dipole magnets. Results obtained for Monte Carlo and real data are presented. Comparison with a method where no matching is used shows a significant reduction of up to $40 \%$ of the fraction of wrongly determined charges.
\end{abstract}

Keywords: Neutrino, OPERA, Drift Tube, Muon Charge Sign, Spectrometer

\section{Introduction}

The OPERA experiment has been designed to observe the direct appearance of $v_{\tau}$ in a $v_{\mu}$ beam by resolving the track left by the short-lived $\tau^{-}$lepton emitted in CC interactions [1]. After analyzing about $90 \%$ of the data five candidate events have been observed [2, 6], corresponding to a significance of $5.1 \sigma$ [6] thanks to the low level and the control of the background. The largest source of background corresponds to charmed particles produced in $v_{\mu} \mathrm{CC}$ interactions, which is suppressed by the identification of the primary (negative) muon. In case the charmed particle decays into a muon, $v_{\mu} N \rightarrow c \mu^{-} X$ with $c \rightarrow$ $\mu^{+} Y$, background may be further reduced by identifying the secondary muon and determining the positive sign of its charge. Therefore identifying either primary or secondary muons is of utmost importance in order to reduce background processes. Moreover about one $v_{\tau} N \rightarrow \tau^{-} v_{\tau} X$ event with $\tau^{-} \rightarrow \mu^{-} \bar{v}_{\mu} \nu_{\tau}$ is expected to be observed in OPERA, assuming full $v_{\mu}-v_{\tau}$ mixing and $\Delta m_{32}^{2}=2.44 \times 10^{-3} \mathrm{eV}^{2}$ [7]. One such candidate has been actually found so far. An efficient and robust estimator of the muon charge sign, in particular, together with a controlled estimation of its error is mandatory on an event-by-event basis.

The use of a magnetic spectrometer enables the measurement of the particle charge and momentum in high energy physics. In the OPERA experiment [8] a cross-sectional area of the order of $70 \mathrm{~m}^{2}$ with a rather uniform field was chosen as a good compromise between needs and costs. The CERN to Gran Sasso (CNGS) muon-neutrino beam [9] has an average energy of $17 \mathrm{GeV}$. A dipolar magnetic field with a uniform magnetization allowing sufficient bending over the whole magnet cross-section was developed and implemented in OPERA. Two large dipoles corresponding to the bi-modular setup were realized. The two magnetized iron arms of each dipole, connected by an upper and a lower yoke, are orthogonal to the neutrino beam [10, 11]. Precision Trackers (PTs) [12] consist of six stations of high precision drift tubes grouped in three pairs, one pair placed in front, one in between and one behind the two magnetized arms. Due to their very low Z-density, each PTs station pair reconstructs straight track segments, enabling the measurement of the deflection angles of the muon trajectories in the curvature plane ${ }^{1}$. A single magnetized arm together with the four adjacent PT-stations defines a Charge Measurement Unit (CMU). Thus the two spectrometers provide four CMUs.

The Kalman filter procedure [14] is used in OPERA for the momentum reconstruction, while the assessment of the charge of the track is based on a independent technique. In the procedure used so far, the deflection is measured independently for each CMU, taking into account the energy losses and the Coulomb scattering [13] when estimating the track momentum. The procedure of the charge-sign determination is referred below as the OPERA Standard Method (OSM) [15], and it is briefly recalled in Appendix A. It relies on the precision on the angles of the two track segments as parts of the muon trajectory at the entry and the exit of the magnet arm. In this paper an up-to-date way to estimate the charge sign is described, by appropriately exploiting the combined information of the CMUs. In the following the new procedure is referred to as the Angular Matching Method (AMM). Results based on Monte Carlo simulations (MC) and real data are presented together with an estimation of the level of impurity, defined as the fraction of muons for which the charge sign is wrongly determined.

It is worthwhile to mention that in the single candidate observed so far in the muonic decay channel of the $\tau$ [4], the low momentum muon stops in the first magnet arm and its charge cannot be measured by the PT system. Instead, the hits recorded by the Resistive Plate Chambers (RPC) that instrument the magnet arm have been used to assess the negative sign of the muon charge with a significance of $5.6 \sigma$. The improvement in algorithms using the PT data for charge-sign determination thus does not affect the analysis of this particular candidate.

\footnotetext{
${ }^{1}$ The OPERA coordinate system is a right-handed Cartesian system, with the $Z$ axis pointing along the horizontal projection of the neutrino beam direction, and the $Y$ axis pointing upward. The bending plane is $Z X$, perpendicular to the vertical magnetic field lines.
} 


\section{The OPERA detector and its muon spectrometers}

The OPERA detector is located at the INFN Laboratori Nazionali del Gran Sasso (LNGS) in Italy [8]. It was exposed between spring 2008 and December 2012 to the CNGS $v_{\mu}$ beam with an average energy of $17 \mathrm{GeV}$ [16], providing a baseline length of about $730 \mathrm{~km}$ to study neutrino oscillation. The contaminations in CC interactions of $\bar{v}_{\mu}, v_{e}$ and $\bar{v}_{e}$ relative to $v_{\mu}$, amount to $2.1 \%, 0.9 \%$ and less than $0.1 \%$, respectively [17]. The prompt $v_{\tau}$ contamination is negligible. The collected data correspond to about $18 \times 10^{19}$ protons on target and a total of 19505 neutrino interactions have been recorded.

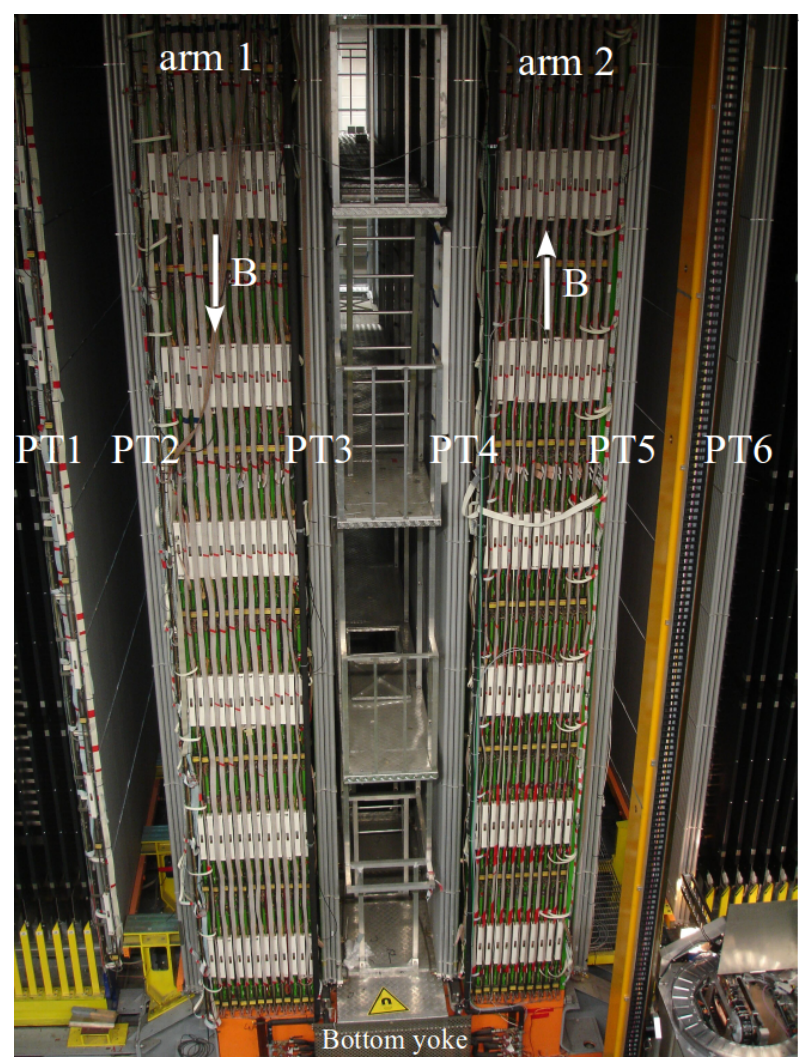

Figure 1: Side view of one of the two OPERA spectrometers.

The OPERA hybrid detector makes use, besides electronic tracking detectors, of nuclear emulsion to observe with unique space resolution the production of $\tau$ leptons in $v_{\tau}$ CC interactions and their subsequent decay within a distance of the order of $1 \mathrm{~mm}$. The topology of the neutrino interactions is recorded in Emulsion Cloud Chamber detectors (ECC) with sub-micrometric spatial resolution. The ECC technique used for the active neutrino target combines nuclear emulsion films and lead plates to meet the requirement of a sub-micrometric precision over a large volume. The active neutrino target is made of 150,000 basic units, called "bricks" and has a total mass of about $1.2 \mathrm{kt}$. The bricks consist of 56 lead plates of $1 \mathrm{~mm}$ thickness interleaved with emulsion films. They are arranged in 62 vertical walls, each of them followed by an electronic Target Tracker (TT) plane made of $X$ and $Y$ plastic scintillator strips having their signal collected by wave-length shifting fibres and read by multi-anode photomultiplier tubes [18]. The TT planes are aimed at triggering the data acquisition and measuring the trajectories of the charged particles through the target, selecting the bricks to be extracted from the walls where neutrino interactions occurred and localizing the area where the scanning of the emulsion films [19] has to start in the search for tracks pointing to the neutrino vertex. The neutrino target is subdivided into two identical Super-Modules (SM).

A large magnetized volume is placed downstream of each SM target. Each spectrometer is an iron dipole, with a uniform magnetic field over a cross-sectional area of $8 \times 8.75 \mathrm{~m}^{2}$. As shown in Figure 1, the magnet consists of two vertical arms with upper and lower horizontal yokes to close the magnetic field circuit. The $1.53 \mathrm{~T}$ magnetic field has opposite directions in the two arms. Non-uniformities have been measured to be less than 3\% [11]. Each magnet arm is segmented in twelve $5 \mathrm{~cm}$ thick iron-slabs, internally instrumented by eleven planes of RPC equipped with $X-Y$ strips for a coarse tracking (spatial resolution $\sim 1 \mathrm{~cm})$. Externally to the arms each spectrometer is equipped by six stations of high precision vertical drift tubes planes, the PTs [12], for precise muon tracking. Each PT station consists of 4 layers of drift tubes. The non-bending coordinate $(Y)$ is coarsely measured by two walls of RPC planes, placed upstream of the magnet with the readout strips inclined by $\pm 42.6^{\circ}$ with respect to the horizontal direction. They are placed $1 \mathrm{~cm}$ upstream and downstream of the first and second drift tube station, respectively.

Each spectrometer consists of two CMUs given by the two pairs of PT stations, upstream and downstream of each magnet arm (Figure 2). The CMUs provide a measurement of the charge and the horizontal projection of the muon momentum, provided the track is reconstructed on both sides of the magnet arm. The spatial resolution of the PT is better than $300 \mu \mathrm{m}$ in the horizontal plane [12, 20].

\section{Monte Carlo simulations}

Two different Monte Carlo simulations based on GEANT3[21] have been used. For both types of simulation muon trajectories have been reconstructed and their momenta evaluated using the full OPERA analysis chain [22].

The first MC simulation (MC-I) was used to demonstrate the performances of AMM as function of the muon momentum. The sample consisted of positive and negative muons emitted at the centre of the targets of either SM1 or SM2, at an angle orthogonal to the drift tubes planes. The momentum was varied between 1 and $100 \mathrm{GeV} / \mathrm{c}$ in steps of $1 \mathrm{GeV} / \mathrm{c}$. The angle is close to those of most muons produced in CNGS CC neutrino interactions in the detector.

The full OPERA simulation chain of the response of the electronic detectors to CNGS neutrinos interactions (MC-II) has been used in a second step in view of evaluating the potential gain that the AMM algorithm may provide in the muon charge determination. It uses the event generator NEGN [23] developed in the framework of the NOMAD experiment [24]. 


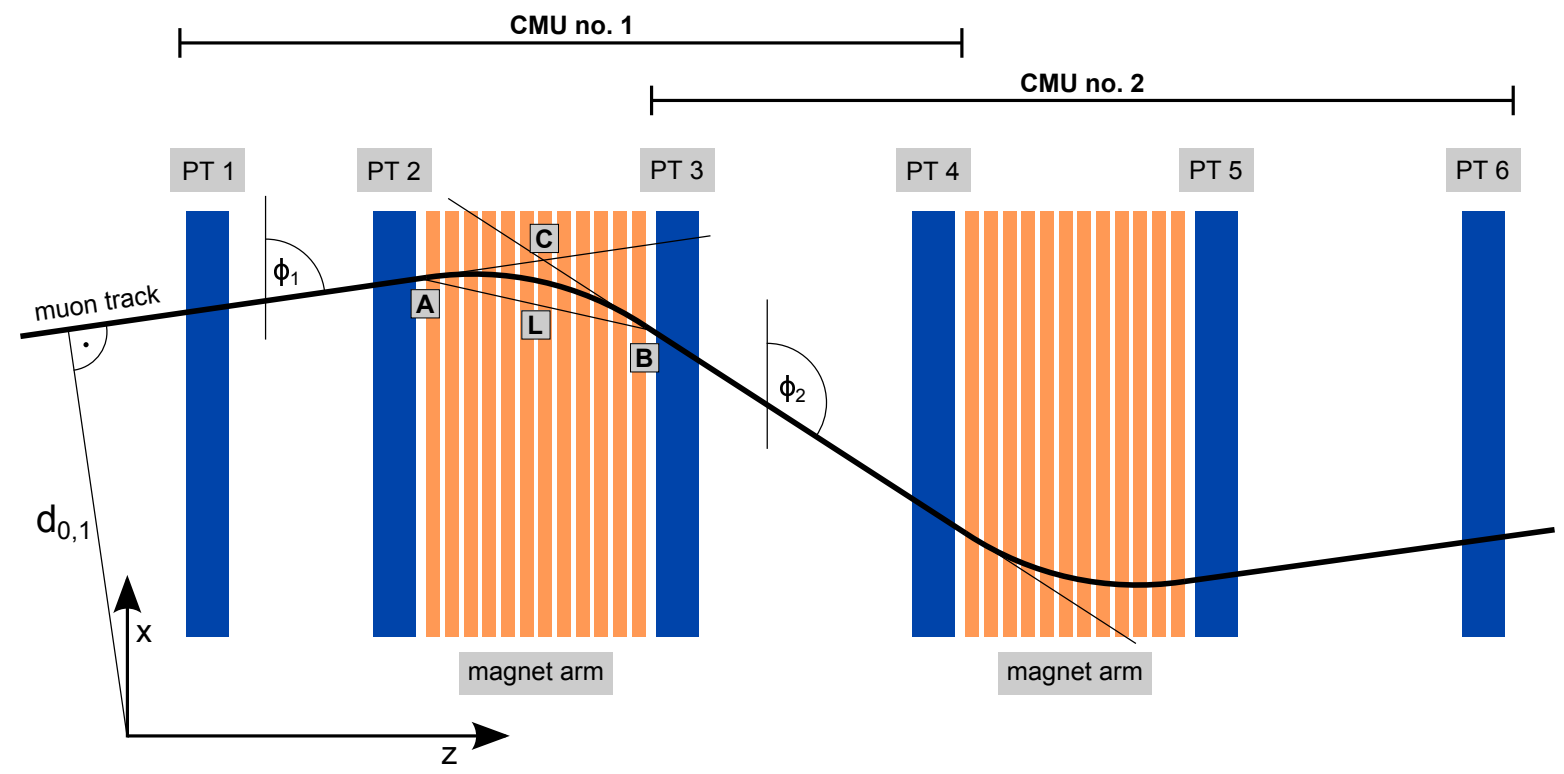

Figure 2: Schematic top view of one of the two OPERA spectrometers. The six Precision Tracker (PT) stations are used for the track reconstruction. Each Charge Measurement Unit (CMU) delivers a measurement of charge/momentum, provided the track is reconstructed on both sides of the magnet arm. PT1-PT4 form the first CMU and PT3-PT6 form the second CMU. The charge sign determination in the first CMU uses the deflection angle $\Delta \phi=\phi_{2}-\phi_{1}$. The OPERA detector has a total of four CMUs, two per spectrometer.

\section{Methodology}

\subsection{Identification of track inconsistencies}

The straight track segments reconstructed by the PT system provide track parameters projected in the horizontal plane $(X$, $Z$ ), orthogonal to the magnetic field lines that are directed along the $Y$ axis. For detailed information on track reconstruction in the PT see [13]. Ideally, two segments on each side $j=1,2$ of a magnet arm are described by the angles $\phi_{j}$ that they enclose with axis $X$ and by their distance $d_{0, j}$ to the reference frame origin (see Figure 2, for reasons of simplicity $d_{0,2}$ is not shown in the figure). $\Delta \phi=\phi_{2}-\phi_{1}$ is the deflection angle. If energy loss and multiple scattering are neglected, a charged particle trajectory in the magnetic field is an arc of a circle tangent to both track segments at their magnet arms entry/exit points (see Figure 3). Calling $\alpha_{j}$ the complement of the angle between the chord joining these two points and track segment $j$, it follows that $\alpha_{1}=\alpha_{2}$ and $\Delta \phi=\alpha_{1}+\alpha_{2}$ (see Appendix B for more details). The relative angular deviation in $\mathrm{CMU}_{i}$

$$
\Delta(\alpha)_{r e l, i}=\left.2 \frac{\alpha_{1}-\alpha_{2}}{\alpha_{1}+\alpha_{2}}\right|_{i}=\left.2 \frac{\alpha_{1}-\alpha_{2}}{\Delta \phi}\right|_{i}
$$

provides a measurement of the mismatch between the two reconstructed track segments. Large values of the mismatch between $\alpha_{1}$ and $\alpha_{2}$ indicate that one or both track segments are reconstructed with poor precision, in which case they may also be wrongly associated, yielding to a wrong muon sign determination.

In the following a weight is constructed such that large angular mismatches result in a small weight.

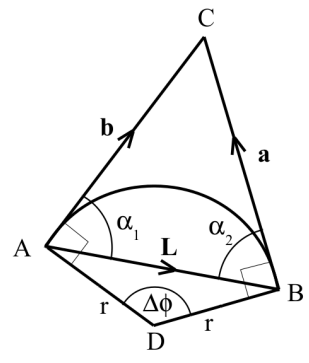

Figure 3: The particle trajectory in the magnetic field is shown (arc of a circle $\mathrm{AB}$ of radius $r$ ). Vectors $\mathbf{a}$ and $\mathbf{b}$ (intersecting at C) are tangents to the circular path at the entry (A) and exit (B) points. A, B and $\mathrm{C}$ refer to points in Figure $2 \Delta \phi=\phi_{2}-\phi_{1}$ is the deflection angle.

The distribution of the reconstructed $\Delta(\alpha)_{\text {rel }}$ for 200,000 negative muons simulated following MC-I (see Section 3) is shown in Figure $4 \mathrm{a}$. It has been fitted with a Voigtian distribution $V(x)$, the convolution of a Gaussian $G(x)$ and a Lorentzian $L(x)$ distribution,

$$
\begin{aligned}
V(x) & =G(x) \otimes L(x) \\
& =A \frac{1}{\sqrt{2 \pi \sigma}} e^{-\frac{1}{2}\left(\frac{x-\mu}{\sigma}\right)^{2}} \otimes \frac{1}{2 \pi} \frac{\gamma}{(x-\mu)^{2}+\frac{1}{4} \gamma^{2}},
\end{aligned}
$$

where $\mu, \gamma$ and $\sigma$ are obtained from the fit. $A$ is a normalization factor. The central value $\mu$ is fully compatible with 0 . The relative error on the angle difference given by the precision on its measurement is well reproduced by a Gaussian distribution except for the long tails that are described by the convolution 


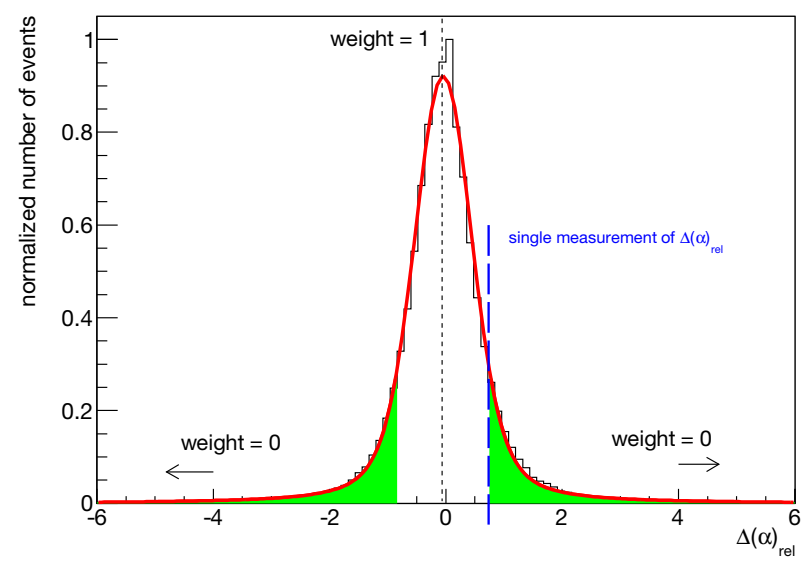

(a) Charge weight principle

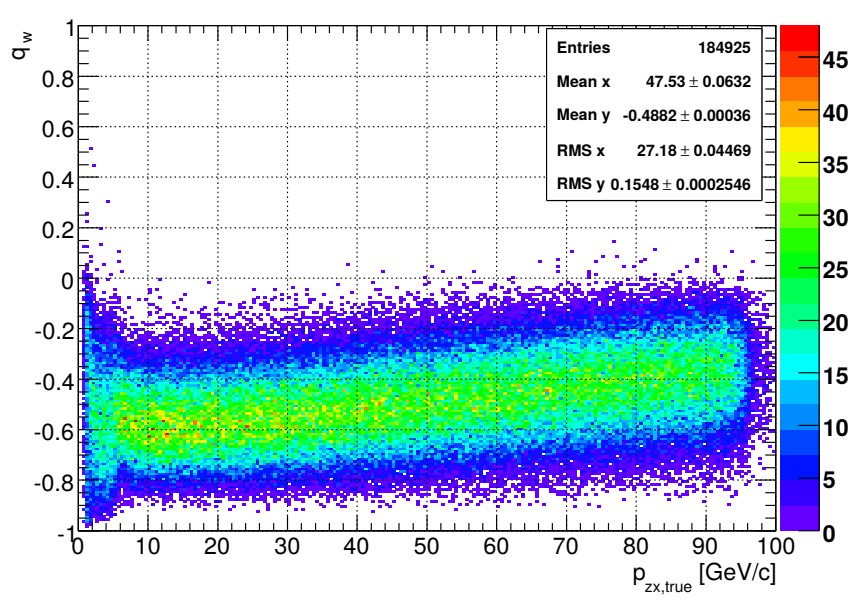

(b) Charge weight dependence on momentum

Figure 4: (a): The black histogram shows the distribution of the relative angular deviations $\Delta(\alpha)_{\text {rel }}($ Eq. 1 ) and the red curve the Voigtian distribution that best fits to it. The central value $\mu$ is indicated by the dashed line. The weight $w_{i}$ attributed to the charge sign measurement obtained in a single $\mathrm{CMU}_{i}$ is given by the integral defined in Eq. (4), (5) and (6). It corresponds to the green area. The smaller $\Delta(\alpha)_{r e l}$ is, the larger is the weight. Figure (b) shows, for simulated $\mu^{-}$, the dependence of the charge weight estimator $q_{w}$ (Eq. (8) ) on the true Monte Carlo momentum $p_{\mathrm{ZX} \text {,true }}$ projected in the horizontal plane. The sign of $q_{w}$ fixes the sign of the reconstructed muon charge (see Section 4.2). For both figures the simulation method MC-I is used with negative muons (see Section 3), with the difference that for Figure (b) the momentum is uniformly distributed between 1 and $100 \mathrm{GeV} / \mathrm{c}$ instead of in steps of $1 \mathrm{GeV} / \mathrm{c}$.

with a Lorentzian distribution. The quality of the track reconstruction in $\mathrm{CMU}_{i}$ is given by its weight

$$
w_{i}=1-\int_{a_{i}}^{d_{i}} V\left(\Delta(\alpha)_{r e l, i}\right) \mathrm{d} \Delta(\alpha)_{r e l, i} .
$$

The integration limits are

$$
\begin{aligned}
& d_{i}=\mu+\left|\Delta(\alpha)_{r e l, i}-\mu\right| \\
& a_{i}=\mu-\left|\Delta(\alpha)_{r e l, i}-\mu\right| .
\end{aligned}
$$

For small values of $\Delta(\alpha)_{\text {rel,i }}$ a weight of $\sim 1$ is attributed to the measurement, while it approaches 0 for large values of $\left|\Delta(\alpha)_{r e l, i}\right|$ (Figure 4a).

\subsection{Charge sign determination}

The OPERA detector consists of four CMU each allowing the determination of the deflection angle $\Delta \phi$. For muons originated in CNGS neutrino interactions, taking into account the polarity $P_{i}$ of the magnetic field in $\mathrm{CMU}_{i}$, the charge sign $c_{i}$ is given by

$$
c_{i}=\frac{\phi_{i 2}-\phi_{i 1}}{\left|\phi_{i 2}-\phi_{i 1}\right|} P_{i}
$$

where $\phi_{i j}$ represents the reconstructed angle in front of $(j=1)$ and behind $(j=2)$ the magnet arm of $\mathrm{CMU}_{i}$. A charge sign estimator is defined as

$$
q_{w}=\frac{1}{n} \cdot \sum_{i=1}^{n} c_{i} \cdot w_{i},
$$

where $n$ denotes the number of used CMUs. The sign of $q_{w}$ corresponds to the reconstructed particle charge sign. Its modulus measures the quality of this determination. Figure $4 \mathrm{~b}$ shows, for simulated $\mu^{-}$, the charge sign estimator $q_{w}$ as a function of the true MC momentum projected in the horizontal plane $p_{\mathrm{ZX}, \text { true }}$. Negative muons with $q_{w}>0$ have their charge misidentified. The momentum dependence of the wrong sign determination is small and further discussed in Section 5. In the range of momentum relevant to the physics of OPERA, this can be an important advantage in comparison with conventional methods in particle physics as these are strongly momentum dependent (e.g. Kalman tracking [14]). The statistically optimal behavior of the Kalman fit, or equivalently a $\chi^{2}$ fit, is actually valid only within linear approximations which may not hold in presence of energy loss and multiple scattering. Our result shows that a non-conventional approach, applied to specific cases and to extract only partial information (charge sign), may be more appropriate. The width of the $q_{w}$ distribution is dominated by the measurement errors on the angles and is essentially momentum independent except at low momentum where the muon trajectory inside the magnets is modified by multiple Coulomb scattering. 


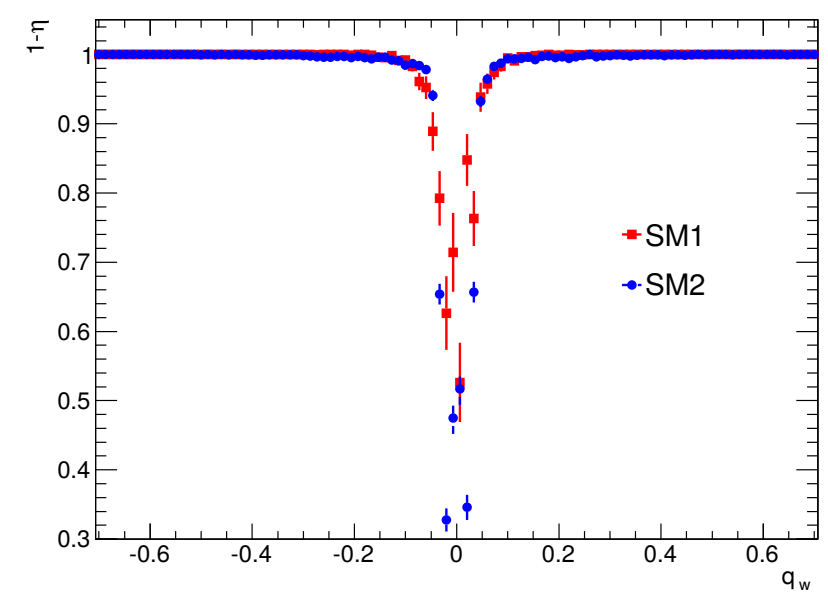

(a)

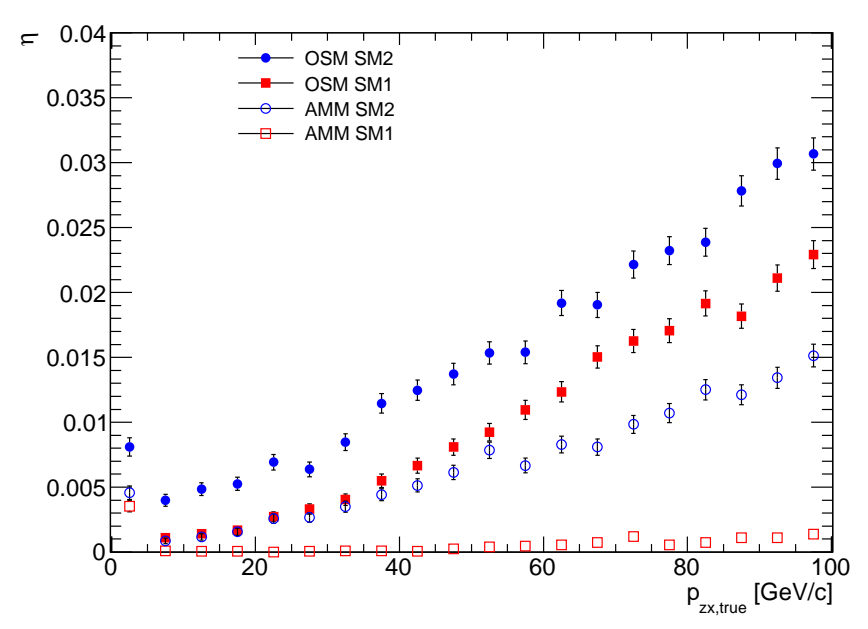

(b)

Figure 5: Purity $1-\eta$ dependence on the estimator $q_{w}$. The vertex of the incoming muons was set in the middle of the target section, in the first (SM1) and in the second supermodule (SM2), respectively. The impurity $\eta$ dependence on the momentum $p_{\mathrm{ZX}, \text { true }}$ is shown in (b). The simulation method is MC-I.

\section{Charge misidentification results}

\subsection{Performances of $A M M$}

To demonstrate the potential of the new charge-sign algorithm the simulation MC-I was used, and the impurity $\eta$ as well as the efficiency $\varepsilon$ for $\mu^{+}$and $\mu^{-}$were calculated. The impurity is defined by

$$
\eta=\frac{n_{w}}{n_{c}}
$$

where $n_{w}$ is the number of wrong charge sign determinations, and $n_{c}$ is the total number of charge sign assignments. The efficiency is defined by $\varepsilon=n / n_{p}$, where $n=n_{c}-n_{w}$ is the number of correct charge sign assignments and $n_{p}$ the number of possible charge sign assignments, where at least one CMU is crossed by the particle. If a cut on the estimator $q_{w}$ is applied the discarded events are not included in $n_{c}$ and $n_{w}$. If no cut is applied to $q_{w}$ then $\eta=1-\varepsilon$.

At very low momentum, purity and efficiency suffer from the multiple Coulomb scattering inside the magnet, and, at very high momentum, from the finite resolution of the PT. A cut on the weight $q_{w}$ increases the purity as shown in Figure 5a at the cost of a reduction of the efficiency. The results obtained for the impurity are shown in Table 1 and in Figure $5 b$ for both OSM and AMM. The following observations are made for AMM:

- Even in the absence of cuts on the weight $q_{w}$, the impurity remains smaller than $0.1 \%$ at momenta larger than 5 $\mathrm{GeV} / \mathrm{c}$, if the vertex is set in the target of SM1. In the momentum range considered in the search for $v_{\tau}$ candidate events, $p_{\mu} i 15 \mathrm{GeV} / \mathrm{c}$, it does not exceed $0.01 \%$ except for momenta smaller than $5 \mathrm{GeV} / \mathrm{c}$ where it increases to $0.4 \%$ (Figure $5 \mathrm{~b}$ ).
- If the vertex is set in the target of SM2 the impurity increases, but still does not exceed $1.6 \%$ at high momentum and $0.2 \%$ for $5 ; p_{\mu} i 15 \mathrm{GeV} / \mathrm{c}$. For $p_{\mu} i 5 \mathrm{GeV} / \mathrm{c}$ it increases to $0.5 \%$ (Figure $5 \mathrm{~b}$ ).

A cut on the weight $\left|q_{w}\right|>0.1$ (or 0.2 ) removes most of the impurities if the vertex is set in the target of SM1 even at very low or very high momentum at the cost of a reduction in the efficiency as large as $10 \%$ at very high momentum. For $5 \mathrm{GeV} / \mathrm{c}<$ $p_{\mu}<15 \mathrm{GeV} / \mathrm{c}$, however, the efficiency remains larger than $99.7 \%$ but it falls to $99 \%$ (or $96 \%$ ) for $p_{\mu} ; 5 \mathrm{GeV} / \mathrm{c}$ when the cut on the weight is applied.

Table 1: Comparison between the total impurities, averaged over the momentum range $1-100 \mathrm{GeV} / \mathrm{c}$, obtained with new AMM and OSM.

\begin{tabular}{ccc} 
Vertex position & $\eta_{O S M}($ all $)[\%]$ & $\eta_{A M M}($ all $)[\%]$ \\
\hline target of SM1 & $1.35 \pm 0.02$ & $0.06 \pm 0.01$ \\
target of SM2 & $1.55 \pm 0.02$ & $0.69 \pm 0.01$ \\
all & $1.45 \pm 0.01$ & $0.37 \pm 0.01$ \\
\hline
\end{tabular}

The already small fraction of wrong charge sign determination obtained with OSM is reduced by an order of magnitude when the vertex is placed in the target of SM1 and by a factor of 4 on average. The potential impact of this improvement on the physics results of OPERA is discussed in Section 7

\subsection{Simulation of CNGS neutrinos CC interactions}

A sample of CNGS beam CC neutrino interactions has been generated in the detector target, using the full OPERA simulation chain MC-II described above [22]. Figure 6] shows the distribution of the product of the reconstructed muon momentum $p_{\mu}$ and its charge in a range extending from $-150 \mathrm{GeV} / \mathrm{c}$ to 100 


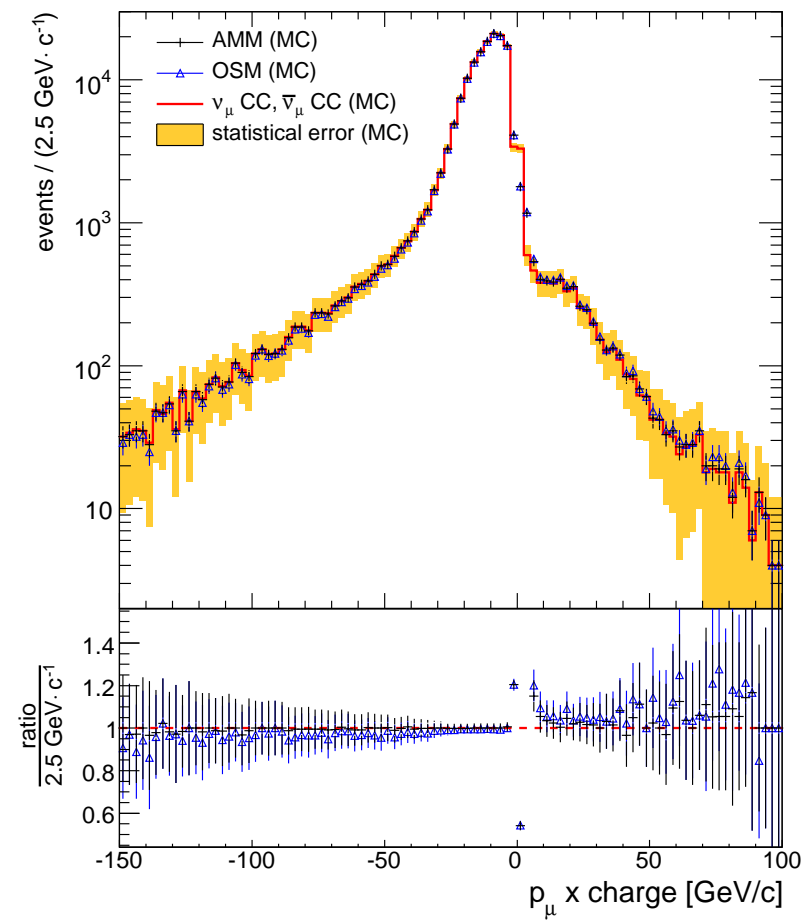

Figure 6: Top: reconstructed (anti-)muon momentum multiplied with the sign of its charge for simulated CNGS CC neutrino interactions (MC-II). The charge is obtained by the truthMC (red histogram), and the charge reconstructed with either OSM (blue histogram with triangle) or AMM (black histogram with vertical mark). For visibility, the statistical error (orange band) on the first spectrum (truth-MC sign) has been multiplied by 3. Bottom: ratios between the truth-MC charge sign and the reconstructed charge signs obtained by either OSM or AMM.

$\mathrm{GeV} / \mathrm{c}$ that includes essentially all events. The fraction of $\mu^{+}$in the total sample of muons estimated with AMM and OSM are $(3.7 \pm 0.1$ (stat.) $) \%$ and $(3.9 \pm 0.1$ (stat.) $) \%$, respectively. The estimated fraction of $\mu^{+}$, mainly emitted in CC interactions of $\bar{v}_{\mu}$ from beam contamination, can only be biased towards values larger than the MC-truth, $(3.4 \pm 0.1$ (stat. $)) \%$. The fraction of incorrect charge assignments is reduced by $\sim 40 \%$, from $0.5 \%$ to $0.3 \%$, with AMM.

Figure 7 shows the muon charge determination impurity as a function of the momentum in the range $2 ; p_{\mu} ; 150 \mathrm{GeV} / \mathrm{c}$ obtained with both methods. If a maximum momentum cut is applied at $15 \mathrm{GeV} / \mathrm{c}$, the impurities obtained with OSM and AMM are respectively $(1.06 \pm 0.04$ (stat.) $) \%$ and $(0.62 \pm 0.03$ (stat.))\%, i.e. a reduction of $\sim 40 \%$. If a cut $\left|q_{w}\right| \dot{i} 0.1$ is applied to the charge determination, the impurity is reduced by a factor of 3 , while the efficiency is reduced to $\sim 86 \%$.

\section{Charge misidentification for real data}

In order to test the new method with real data, two investigations were carried out.

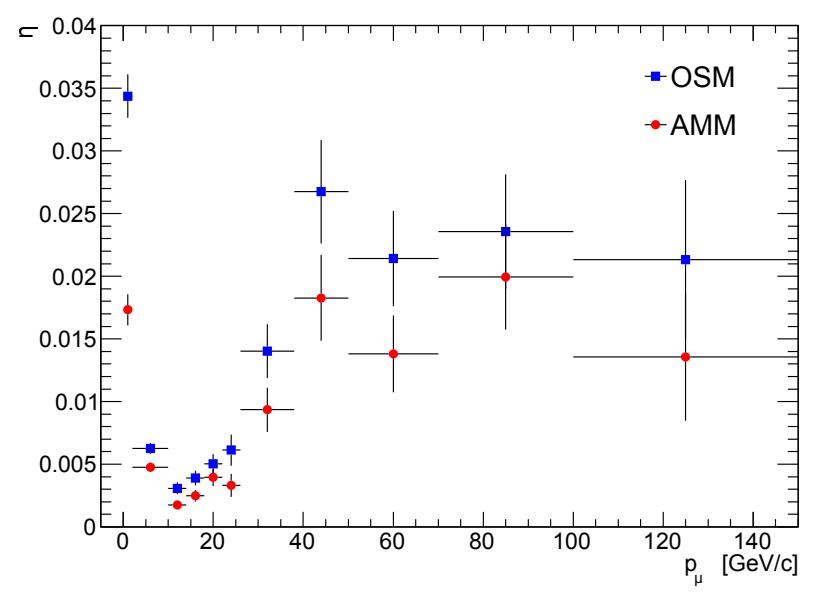

Figure 7: Dependence on the muon momentum $p_{\mu}$ of the impurity $\eta$ on the charge sign determination for simulated CNGS $\mathrm{CC}$ neutrino interactions (MC-II).

Neutrino interactions were selected in which the muon propagates through both spectrometers (number of used CMU $\left.n_{C M U}=4\right)$ and has one charge measurement in disagreement with the other three measurements. The single sign measurement is more likely to be incorrect than the other three measurements. Figure 8 shows as an example the weight of the three stations in agreement $q_{w, a}$ and the weight of the one station in disagreement, $q_{w, d}$. Compared to $\left|q_{w, a}\right|,\left|q_{w, d}\right|$ is generally very small, $\left|q_{w, d}\right|<0.2$.

The aim of the second investigation is to estimate the impurity, $\eta_{\mathrm{SM}}$, for muons crossing one complete spectrometer $\left(n_{\mathrm{CMU}}=\right.$ $2)$. For that purpose, the data sample with particles crossing both spectrometers is used $\left(n_{\mathrm{CMU}}=4\right)$. It has been verified that all four CMU have equal systematics [20, 25] and therefore an equal impurity $\eta_{\mathrm{SM}}$ is expected for both spectrometers. The fraction of tracks with a different charge sign measurement in both spectrometers is then given by

$$
\frac{n^{+-}}{n}=2 \eta_{\mathrm{SM}}\left(1-\eta_{\mathrm{SM}}\right)
$$

where $n$ is the total number of muons and $n^{+-}$is the number of events where different charge signs are obtained. The charge sign is reconstructed independently in each spectrometer. Figure 9 shows the impurity obtained by this method in the muon charge determination, as a function of momentum, for real data and for MC predictions. The impurity is kept below $0.5 \%$ and the muon charge is correctly determined with an efficiency larger than $99.5 \%$ for momenta below $15 \mathrm{GeV} / \mathrm{c}$, the momentum range relevant for the study of $v_{\mu} \rightarrow v_{\tau}$ oscillations (Figure 9a). An additional cut at $\left|q_{w}\right|>0.1$ allows a further reduction of the impurity at the cost of an increase of the fraction of sign indetermination (Figure 9b). This result is relevant for most of the OPERA events as in $80 \%$ of the CC interactions, the muon crosses at least one full spectrometer. In $35 \%$ of the cases both spectrometers are crossed allowing a further reduction of the impurity. 


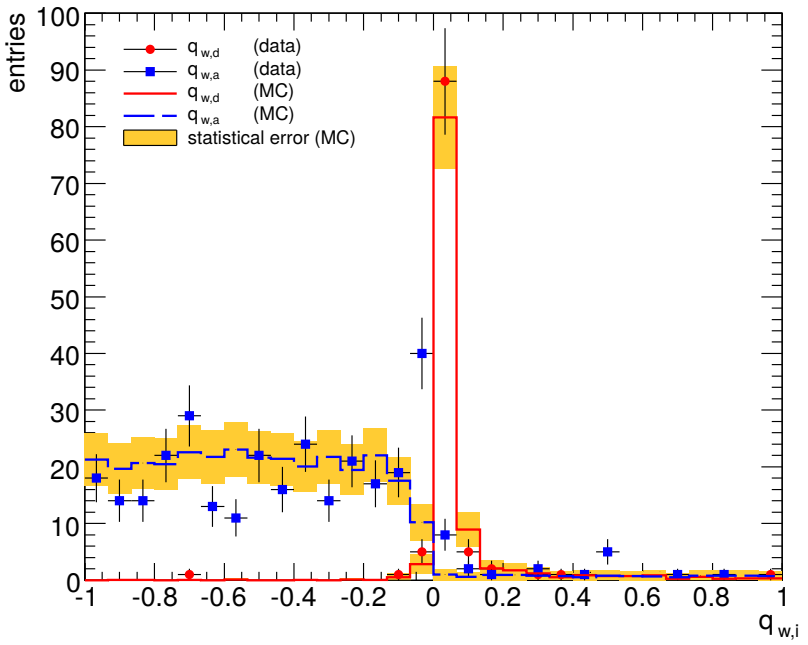

Figure 8: Real data and MC comparison: Tracks that propagate through the all four charge measurement units and where three stations are in agreement and one station is in disagreement are used. $q_{w, a}$ is the weight of the stations in agreement while $q_{w, d}$ is the weight of the station in disagreement. This station is expected to have more often delivered a wrong charge sign, and indeed it yields a small absolute value of the weight.

\section{Conclusion and outlook}

A new method (AMM) has been developed in the framework of the OPERA experiment to improve the determination of the muon charge sign in the spectrometers. In each CMU a weight is assigned to the matching between the measured angles of the two straight track segments at the entry and exit of the magnet arm, projected in the plane of curvature. These weights are then combined to compute a charge sign estimator, the sign of which determines the charge sign of the muon. Its modulus measures the quality of this determination. A lower cut applied to the weight improves the purity - the fraction of correct charge sign determinations - at the cost of some reduction in the efficiency, the fraction of muons for which the charge is determined. The purity naturally increases with the number of CMUs that are crossed by the muon. It has only a small momentum dependence and is affected by the two irreducible effects: a) at small momentum, the multiple Coulomb scattering suffered by the muon inside the magnet, and b) at high momentum and small deflection, the finite resolution in the measurement of the track segment angles.

AMM has been used to evaluate the purity in the charge sign measurement by one spectrometer for real CNGS beam data by comparing how often both spectrometers measure the same sign. The impurity is kept below $0.5 \%$ in the momentum range relevant for the OPERA main analysis.

AMM analysis is part of a campaign of studies to reduce the backgrounds for the OPERA experiment. The estimation of the large-angle muon scattering background which was formerly

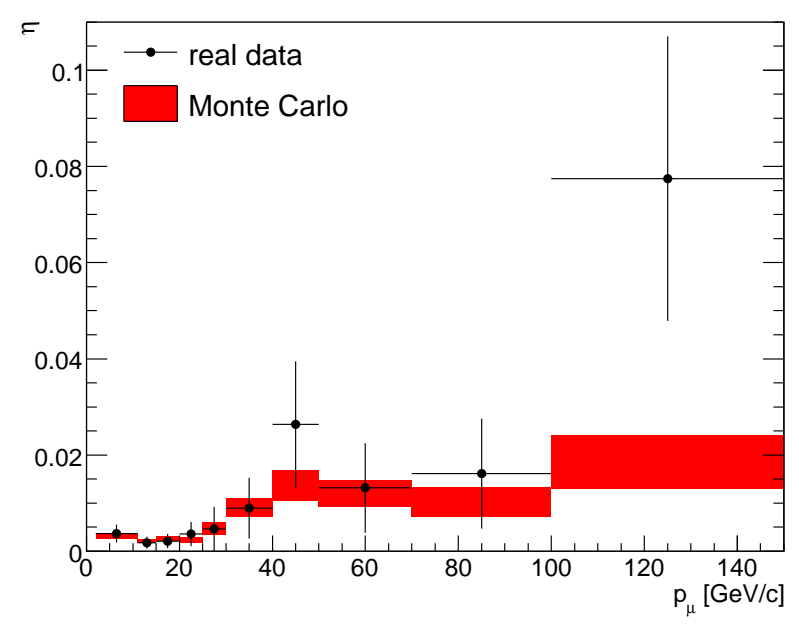

(a) No cut.

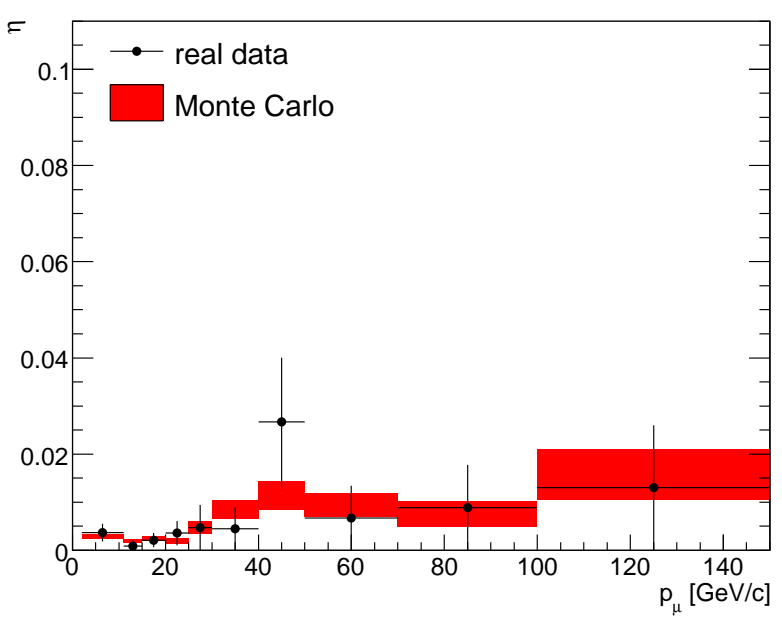

(b) Cut at $\left|q_{w}\right|>0.1$

Figure 9: Evaluation of the impurity $\eta$ with real data compared to the MC expectation as a function of the muon momentum $p_{\mu}$ for one SM using AMM (Eq. 8). The red band corresponds to the $\pm 1 \sigma$ statistical uncertainty. The simulation method is $\mathrm{MC}-$ II. In Figure 9a no cut is applied on the weight. In Figure 9b a cut is applied at $\left|q_{w}\right|>0.1$.

based on conservative assumptions has been recently updated[26] and reduced to a level where charm decays now contribute for about $95 \%$ of the background in the muonic channel. This allows for a more significant role of the AMM algorithm in improving the sensitivity of the experiment.

The use of AMM is not, a priori, restricted to the configuration of the OPERA spectrometers and could be adapted to other experiments, for example to decrease the fraction of $\mathrm{CC}$ events with wrong muon charge sign determination or to better control the systematics in the separation between $v_{\mu}$ and $\bar{v}_{\mu} \mathrm{CC}$ interactions. Experiments with point-like track measurements in the magnetic field (e.g. [27]) may adapt the method for their purposes by using three points to form the triangle shown in 
Figure 3 instead of two tangents.

\section{Appendix A}

The OSM used so far by the OPERA experiment for the charge sign determination is described in detail in [15]. In each $\mathrm{CMU}_{i}$ a weight $w_{i}$ is computed that takes account the measurement precision of the angles $\phi_{1}$ and $\phi_{2}$ made by the two track segments with the transverse direction $\mathrm{X}$ in the horizontal plane of projection, the plane of curvature,

$$
w_{i}=\frac{\phi_{2}-\phi_{1}}{\sqrt{\sigma_{\phi_{1}}^{2}+\sigma_{\phi_{2}}^{2}}}
$$

If all signs are equal, that sign represents the result. The weights of each measurement are added in quadrature to form a global weight for the final result. If the signs differ, the sign measured by the majority of the CMU is used. Only the weights of the stations belonging to the majority are used and added up for the output. If an equal number of positive and negative signs is obtained, it is assumed, that the measurement is disturbed by the presence of additional hits due to the leakage of the tail of the hadronic and electromagnetic showers from the target. Since showers are rapidly absorbed by the magnet, the sign from the CMU closest to the main event vertex is rejected.

\section{Appendix B}

The quantities defined at the beginning of Sec. 4.1 satisfy the Hessian normal form (see also Figures 2 and 3 )

$$
\left(\left(\begin{array}{c}
x_{j} \\
z_{j}
\end{array}\right)-d_{0, j}\left(\begin{array}{c}
\sin \phi_{j} \\
-\cos \phi_{j}
\end{array}\right)\right) \cdot\left(\begin{array}{c}
\sin \phi_{j} \\
-\cos \phi_{j}
\end{array}\right)=0
$$

$\Delta \phi=\left|\phi_{2}-\phi_{1}\right|$ being the deflection angle. If energy loss and multiple scattering are neglected the charged particle trajectory in the magnetic field is an arc of a circle tangent to both track segments at their magnet entry/exit points.

One defines the quantities projected in the bending horizontal plane:

- $\mathbf{L}$, a direction vector of the line connecting $A$, the entry point of the upstream segment in the magnet and $B$, the exit point of the downstream segment.

- $\mathbf{a}, \mathbf{b}$, a direction vector along the upstream segment and a direction vector opposite to the downstream segment, respectively,

If follows that

$$
\begin{aligned}
\mathbf{b} & =\left(\begin{array}{c}
z_{2}-z_{1} \\
x_{1}\left(z_{2}\right)-x_{1}\left(z_{1}\right)
\end{array}\right) \\
\mathbf{a} & =\left(\begin{array}{c}
z_{1}-z_{2} \\
x_{2}\left(z_{1}\right)-x_{2}\left(z_{2}\right)
\end{array}\right) \\
\mathbf{L} & =\left(\begin{array}{c}
z_{2}-z_{1} \\
x_{2}\left(z_{2}\right)-x_{1}\left(z_{1}\right)
\end{array}\right),
\end{aligned}
$$

where $x_{j}\left(z_{i}\right)$, defined by Eq. $\mathrm{B} .1$, is the $\mathrm{X}$ coordinate of the intersection point of track segment $j=1,2$ with respectively the front face of the magnet arm at $z=z_{1}$ and the back face at $z=z_{2}$. It also follows

$$
\begin{aligned}
& \alpha_{1}=\arccos \left(\frac{\mathbf{b} \cdot \mathbf{L}}{b \cdot L}\right) \cdot s_{1} \\
& \alpha_{2}=\arccos \left(-\frac{\mathbf{a} \cdot \mathbf{L}}{a \cdot L}\right) \cdot s_{2},
\end{aligned}
$$

where signs $s_{1,2}$ are given by

$$
s_{1,2}=\frac{x_{1,2}\left(z_{2,1}\right)-x_{2,1}\left(z_{2,1}\right)}{\left|x_{1,2}\left(z_{2,1}\right)-x_{2,1}\left(z_{2,1}\right)\right|}
$$

In order to calculate both angles $\left(\alpha_{1}\right.$ and $\left.\alpha_{2}\right)$, one uses the second parameter of the track segment fit $\left(d_{0}\right)$, usually neglected for the charge sign determination. Assuming a perfect circular trajectory in the magnet (no energy loss, no multiple Coulomb scattering, homogeneous field) and infinite measurement precision, one get $\alpha_{1}=\alpha_{2}$ and $\Delta \phi=\alpha_{1}+\alpha_{2}$.

\section{Acknowledgements}

We thank CERN for the successful operation of the CNGS facility and INFN for the continuous support given to the experiment through its LNGS laboratory. We acknowledge funding from our national agencies: Fonds de la Recherche ScientifiqueFNRS and Institut Interuniversitaire des Sciences Nucléaires for Belgium, MoSES for Croatia, CNRS and IN2P3 for France, BMBF for Germany, INFN for Italy, JSPS, MEXT, QFPU (Global COE programme of Nagoya University) and Promotion and Mutual Aid Corporation for Private Schools of Japan for Japan, SNF, the University of Bern and ETH Zurich for Switzerland, the Russian Foundation for Basic Research (grant 12-02-12142 ofim, 15-02-01056_a), the Program for the Support of Leading Scientific School, contract SS 3110.2014.2, the Presidium of the Russian Academy of Sciences basic research program Fundamental Properties of Matter and Astrophysics, and the Ministry of Education and Science of the Russian Federation for Russia, and the National Research Foundation of Korea Grant No. 2011-0029457 for Korea. The authors would also like to thank Laura Vanhoefer for fruitful discussions and comments. B.B. and M.M. would also like to thank Ann Fielding for language support.

\section{References}

[1] M. Guler, et al. (OPERA Collaboration), Experiment proposal, CERNSPSC-2000-028.

[2] N. Agafonova, et al. (OPERA Collaboration), Observation of a first $v_{\tau}$ candidate event in the OPERA experiment in the CNGS beam, Phys. Lett. B 691 (2010) 138-145.

[3] N. Agafonova, et al. (OPERA Collaboration), Search for $v_{\mu} \rightarrow v_{\tau}$ oscillation with the OPERA experiment in the CNGS beam, New J. Phys. 14 (2012) 033017. doi:10.1088/1367-2630/14/3/033017

[4] N. Agafonova, et al. (OPERA Collaboration), Evidence for $v_{\mu} \rightarrow v_{\tau}$ appearance in the CNGS neutrino beam with the OPERA experiment, Phys.Rev. D89 (2014) 051102. doi:10.1103/PhysRevD.89.051102 
[5] N. Agafonova, et al. (OPERA Collaboration), Observation of tau neutrino appearance in the CNGS beam with the OPERA experiment, PTEP 2014 (10) (2014) 101C01. doi:10.1093/ptep/ptu132

[6] N. Agafonova, et al. (OPERA Collaboration), Discovery of $\tau$ Neutrino Appearance in the CNGS Neutrino Beam with the OPERA Experiment, PRL 115 (2015) 121802. doi:10.1103/PhysRevLett.115.121802

[7] K. Olive, et al., Review of Particle Physics, Chin.Phys. C38 (2014) 090001. doi:10.1088/1674-1137/38/9/090001

[8] R. Acquafredda, et al. (OPERA Collaboration), The OPERA experiment in the CERN to Gran Sasso neutrino beam, JINST 4 (2009) P04018. doi : 10.1088/1748-0221/4/04/P04018

[9] G. Acquistapace et al., The CERN neutrino beam to Gran Sasso (NGS): Conceptual technical design, CERN-YELLOW-98-02; http://proj-cngs.web.cern.ch/proj-cngs/.

[10] M. Ambrosio et al., The OPERA magnetic spectrometer, IEEE Trans. Nucl. Sci. 51 (2004) 975.

[11] A.Cazes et al., Electromagnetic characterization of the 990-ton gapless magnets for the OPERA experiment, JINST 2 (2007) T03001, Nucl.Phys.Proc.Suppl. 172 (2007) 165.

[12] R. Zimmermann, et al., The precision tracker of the OPERA detector, Nucl. Instrum. Meth. A555 (2005) 435. doi:10.1016/j.nima. 2005. 09.003

[13] B. Wonsak, Die Spurrekonstruktion für das Driftröhren-MyonSpektrometer des Neutrino-Experimentes OPERA Ph.D. thesis, University of Hamburg, (in German) (2007).

URL https://www-opera.desy.de/publications/ Doktorarbeit-Bjoern-Wonsak.pdf

[14] R. Kalman, A new Approach to Linear Filtering and Prediction Problems, Journal of Basic Engineering 82.

[15] R. Zimmermann, Charge Sign Determination with the Precision Tracker of OPERA OPERA public note 105 (2009).

URL http://operaweb.lngs.infn.it/Opera/publicnotes/ note105.pdf

[16] R. Bailey, et al., CERN-SL-99-034-DI, INFN/AE-99/05 (1999) addendum to G. Acquistapace et al., CERN-98-02, INFN/AE-98/05 (1998). URL https://proj-cngs.web.cern.ch/proj-cngs/PDF_files/ sl-99-034.pdf

[17] N. Agafonova, et al. (OPERA Collaboration), Limits on muon-neutrino to tau-neutrino oscillations induced by a sterile neutrino state obtained by OPERA at the CNGS beam, JHEP, 06, (2015) 069. doi:10.1007/ JHEP06 (2015) 069.

[18] T. Adam et al., The OPERA experiment target tracker, Nucl. Instrum. Meth. A 577 (2007) 523.

[19] N. Agafonova et al. (OPERA Collaboration), Procedure for short-lived particle detection in the OPERA experiment and its application to charm decays, Eur. Phys. J. C 74, 2986 (2014).

[20] N. Agafonova, et al. (OPERA Collaboration), Measurement of the atmospheric muon charge ratio with the OPERA detector, Eur. Phys. J. C67 (2010) 25-37. doi:10.1140/epjc/s10052-010-1284-8

[21] R. Brun, et al., Technical report CERN-DD/EE/84-1 (1987). URL http://wwwasd . web.cern.ch/wwwasd/geant/

[22] N. Agafonova, et al. (OPERA Collaboration), Study of neutrino interactions with the electronic detectors of the OPERA experiment, New J Phys. 13 (2011) 053051. doi:10.1088/1367-2630/13/5/053051

[23] D. Autiero, The OPERA event generator and the data tuning of nuclear re-interactions, Nucl. Phys. Proc. Suppl. 139 (2005) 253-259. doi:10. 1016/j.nuclphysbps.2004.11.168

[24] J. Altegoer, et al., The NOMAD experiment at the CERN SPS, Nucl. Instrum. Meth. A404 (1998) 96-128. doi:10.1016/S0168-9002(97) 01079-6

[25] N. Agafonova, et al. (OPERA Collaboration), Measurement of the $\mathrm{TeV}$ atmospheric muon charge ratio with the complete OPERA data set, Eur.Phys.J. C74 (7) (2014) 2933. doi:10.1140/epjc/ s10052-014-2933-0

[26] A. Longhin, A. Paoloni, F. Pupilli, Large-angle scattering of multi-GeV muons on thin Lead targets, doi : 10.1109/TNS . 2015. 2473674 (2015), arXiv: 1506.08759.

[27] The Reach of INO for Atmospheric Neutrino Oscillation Parameters, T. Thakore, A. Ghosh, S. Choubey, and A. Dighe, JHEP 1305 (2013) 058; Physics Potential of the ICAL detector at the India-based Neutrino Observatory (INO) S. Ahmed et al (ICAL Coll.). arXiv:1505.07380; The 